\title{
Wilderness Therapy as a Specialized Competency
}

\author{
Parker D. Houston \\ Department of Graduate Psychology, Azusa Pacific University \\ P.O. Box 7000, Azusa, CA 91702, U.S.A \\ Tel: 1-626-815-6000Ｅ-mail: phouston@apu.edu \\ Joshua J. Knabb \\ Department of Graduate Psychology, Azusa Pacific University \\ P.O. Box 7000, Azusa, CA 91702, U.S.A
}

Tel: 1-626-815-6000Ｅ-mail: jknabb@apu.edu

Robert K. Welsh

Department of Graduate Psychology, Azusa Pacific University

P.O. Box 7000, Azusa, CA 91702, U.S.A

Tel: 1-626-815-6000 E-mail: rwelsh@apu.edu

Beth M. Houskamp

Department of Graduate Psychology, Azusa Pacific University

P.O. Box 7000, Azusa, CA 91702, U.S.A

Tel: 1-626-815-6000Ｅ-mail: bhouskamp@apu.edu

David Brokaw

Department of Graduate Psychology, Azusa Pacific University

P.O. Box 7000, Azusa, CA 91702, U.S.A

Tel: 1-626-815-6000Ｅ-mail: bhouskamp@apu.edu

\begin{abstract}
Wilderness Therapy (WT) is an emerging psychotherapeutic intervention for the treatment of youth and other populations and is unique in that it is a competency area of psychology that differs from traditional psychotherapy. Because of this, clinical training received in doctoral psychology programs may not be sufficient to ensure competent practice of wilderness therapy. The goal of this article was to address the need for standardization and the development of core competencies for WT as a specialty area. Furthermore, because WT is a specialty area, this article proposes an outline of key competencies for psychologists looking to practice within the field of WT. Given that the majority of programs serve adolescent populations, the competencies outlined in this article will be specifically targeted at this population.
\end{abstract}

Keywords: Wilderness therapy, Core competencies, Adventure therapy, Adventure-based counseling

\section{Introduction}

Wilderness Therapy (WT) has recently emerged as a promising therapeutic treatment. In spite of this, the variables that contribute to its success are not well understood. Moreover, in the last decade, WT has received added attention as evidenced by several meta-analyses published in the field (Gillis \& Casson, 1994; Hans, 1997, 2000; Hattie, Marsh, Neill, \& Richards, 1997; Marsh, 1999; Neill, 2003; Wilson \& Lipsey, 2000). Although these studies lend support to the notion that WT has been increasingly recognized as a legitimate psychotherapeutic intervention and demonstrate that professionals in the WT field are attempting to consolidate and appreciate the mechanisms responsible for WT's efficacy, conflicting results from, and problems with, these 
studies have surfaced (Hattie, Marsh, Niell, \& Richards, 1997). For example, Newes (2001) suggests that problems associated with standardization remain among the existing meta-analyses. Fundamentally, uncertainty lingers as to exactly what techniques and standardization practices are being utilized in WT programs. The central objective of this article was to develop a set of core competencies for psychologists wanting to practice in a WT setting in order to allow for standardization of the vast number of WT programs currently available.

WT is unique in that it creates a microcosm of difficulties experienced by clients in a manageable environment to help clients build self efficacy, patience, and problem solving skills (Nadler \& Luckner, 1992). WT immerses youth in an unfamiliar environment in which they often live with peers, participate in individual and group psychotherapy under licensed professionals, and learn skills that parallel real life struggles with the hope that they will incorporate these new skills into their repertoire when they return to their families (Russell, 2003). Since most therapeutic endeavors work to improve comfort for the client, this aspect makes WT especially distinct in that it utilizes perceived risk in the therapeutic paradigm (Fletcher \& Hinkle, 2002).

Despite the promise WT holds, WT psychologists have not been able to agree upon a definition of exactly what it is that they do (Berman \& Davis-Berman, 2001). Moreover, although WT psychologists must obtain a license in order to practice professional psychology, specific WT principles and preparation are absent from the general licensing procedures, leaving the licensed psychologist ill-equipped to enter the field of WT. Ultimately, additional specified criteria for these psychologists may protect the public from psychologists who are not uniquely trained in principles of WT. The challenge at hand is for WT to move towards a professional standard of care without losing the creative outreach that has previously defined adventure and wilderness approaches (Berman \& Davis-Berman, 2001).

Furthermore, WT research must discover the common therapeutic components of programs that seem most successful and establish industry standards for psychologists to implement. The creation of core competencies for WT programs will provide a foundation from which to base future training of WT psychologists and the institution of programs. In agreement, Neill (2003) suggests that there is considerable room for improvement in developing more effective practices and achieving stronger outcomes. Moreover, recent projects have sought to examine the factors that may be related to the variability between programs (see Russell, 2003). Because of this, it has become increasingly important for WT programs to adopt a standard set of core competencies.

Several potential benefits exist in establishing core competencies for WT practitioners. First, professional psychologists who would like to enter the field of WT will have literature to turn to in order to establish a basic fund of knowledge. This will enable new WT psychologists to feel more competent or grounded in sound practice. Second, new programs can have greater confidence in their quality based on agreed-upon standards of training. Improved standards may also strengthen the objectivity with which consumers and third party payers can more accurately evaluate programs. Finally, increased respect and support from managed care and families in crisis may increase the population served by WT (Newes, 2001).

In spite of the aforementioned benefits of competencies for WT, much like other areas that have later become distinct competencies, the explosive and rather unregulated growth of WT programs has precluded a set of guidelines for what it means to be a WT psychologist (Brotman, Liberi, \& Wasylyshyn, 1998). Without a minimum set of standards that allow for competent practitioners, it is unlikely that WT will improve its reputation as a compelling treatment (Niell, 2003). A shift towards WT competencies may allow for a more scholarly movement in the field in that professionals involved in WT would actually promote accountability (Priest, 2001). Moreover, competency is an ethical standard in the discipline of psychology (see APA Ethics Code, 2003). The APA (2003) maintains, "In those emerging areas in which generally recognized standards for preparatory training do not yet exist, psychologists nevertheless take reasonable steps to ensure the competence of their work and to protect clients from harm." With the current lack of guidelines for WT psychologists, psychologists in the field of WT may not be able to uphold the current standards of competency set forth by APA.

Despite the lack of WT competencies, many other areas of specialization allow for competencies within the profession of psychology (Roe, 2002). For example, child custody proceedings, forensic psychology, executive coaching, and a myriad of other specialties provide competencies (APA). As these models provide examples of APA models of specialization, they may offer a useful framework for further developing core competencies for WT. And although WT may not meet full APA criteria for a recognized specialization, this article will attempt to use the strictest criteria for evaluating WT as a distinct competency in order to provide an amalgam of useful core competencies for WT to move forward towards a more standard level of care. 


\section{Definition of key terms}

At this point, it is important to explain the distinction between the many different terms used to describe WT. Because of the plethora of differing terms, authors have suggested that one of the dilemmas facing the WT field is a lack of common language and jargon (Gass, 1993). Wilderness therapy (WT) is often referred to as adventure-based counseling (ABC), adventure therapy (AT), and more recently, Outdoor behavioral healthcare (OBHC). These emerging alternative treatments help clients overcome problems with addiction, facilitate adjustment, and develop psychological and emotional health (Russell, 2000). These four key terms can be found in the literature and reflect some confusion about categories of programs. However, WT programs are only a small part of the larger wilderness experience program (WEP) industry, consisting of about 38 programs compared to 500 in the larger category (Friese, Hendee, \& Kinziger, 1998). These WEP programs are organizations that conduct outdoor programs in wilderness or comparable lands for purposes of personal growth, therapy, rehabilitation, education, or leadership/organizational development. More than 700 potential WEPs were identified through a search of multiple sources and then surveyed, with promotional materials and response forms received from 70 percent of them (Friese, Hendee, \& Kinziger, 1998). Most of the early educational programs, such as Outward Bound (OB)(a type of WEP), have laid the foundation for the newer programs that tend to include a more intentional and specific psychotherapeutic component (Nassar-McMillan \& Cashwell, 1997).

\section{Origins and history of WT programs}

The earliest roots of wilderness therapy date back over a century and can be traced to Europe in the late 1800s (Nassar-McMillan \& Cashwell, 1997). Within the United States, however, WT can be traced back to 1901 (Fletcher \& Hinkle, 2002) and has been consistently implemented for nearly 60 years (Mcnamara, 2002). Organized programs such as Outward Bound were introduced in the United States in the 1960s after their inception in Europe in 1941(Fletcher \& Hinkle, 2002). For example, Outward Bound (OB) is an international, non-profit, independent educational organization with approximately 40 schools around the world. Outward Bound programs aim to foster the personal growth and social skills of participants by using challenging expeditions in the outdoors. In addition to Outward Bound, Project Adventure was developed in the 1970s to combat some of the obstacles observed with Outward Bound such as cost, intensity, and duration (Schoel, Prouty, \& Radcliffe, 1988). Moreover, several wilderness experience programs came out of Brigham Young University (BYU) in Provo, Utah in the 1960s. BYU made available outdoor survival classes and eventually established primitive living courses throughout the United States and Canada, developing the 26-day wilderness survival course that became the signature of the Boulder Outdoor Survival School (Friese, Hendee, \& Kinziger, 1998). Among these programs were the roots for Aspen Achievement Academy, now regarded as one of the top WT programs. The Aspen Achievement Academy is a licensed treatment program that integrates an accredited academic component, a sophisticated therapeutic model, and an experiential education curriculum in a healing wilderness environment. The academy creates a modern "rite of passage" designed to assist adolescents in making the transition to responsible young adulthood. It guides each participant through a series of powerful metaphors that symbolize stages of growth, provide deeper insight towards developing self-reliance, and generate a sense responsibility for self and community. Aspen's clinical focus is on insight-oriented therapy, rather than behavior modification (Aspen Academy, 2006).

Although numerous other wilderness programs were established during the $80 \mathrm{~s}$ and $90 \mathrm{~s}$, most adventure programs are based on the Outward Bound model (Bandoroff, 1992; Hattie, Marsh, Niell, \& Richards, 1997; Russell, 2001). Moreover, much theoretical history for WT programs is based on Native American ceremonial rites of passage into adulthood intended to foster solitude, differentiation, and responsibility (Beringer, 2004).

\section{Foundations of WT}

Both wilderness and adventure theory have developed out of experiential learning theories (Fletcher \& Hinkle, 2002). The experiential learning process is the foundation of WT, which emphasizes the acquisition of life skills through challenge activities and the transfer of those skills into real life situations (Nadler \& Luckner, 1995). In these situations, clients are essentially learning by doing (Gass, 1993). Experiential learning involves the stages of experience, reflection, processing, and life application (Nadler \& Luckner, 1992). Nadler and Luckner (1992) suggest that adolescents in WT programs may also go through a "disequilibrium," or breaking period, in their first stages of treatment. During this phase, participants are confronted with the reality that their current patterns of functioning are no longer adaptive for the given situation (SUWS, personal communication, March 22, 2005). Thus, clients are forced to take a new, and hopefully more adaptive, approach to their problems. This basic theory is a central aspect of many modern WT programs. 
The Accreditation Council of the Association for Experiential Education lists five core principles of experiential learning philosophy (Leemon, Place, Ajango, \& Wood, 2005). First, adventure activities are selected and used in accordance with the organization's mission statement, goals for instruction, and intended outcomes. This, meaning that learning occurs when carefully chosen experiences or activities are used intentionally to promote learning, personal growth, or positive change. Second, activities are designed and conducted in such a way as to engage the learner in direct experience. For instance, each adventure activity should be created and conducted in a manner that allows clients to take part in as many aspects of the activity as possible. Third, participants are encouraged to be personally involved, and responsible for their own learning since experiential learning is an active rather than passive process, with participants motivated by, responsible for, and accountable for their own learning and growth. Fourth, appropriate education methods are used to enable participants to develop new knowledge from their experiences. This process can be achieved in a number of ways, including dialogue, group discussion, writing, and drawing. Lastly, activities are made meaningful through the use of natural consequences. Natural consequences are those that arise from an action or inaction (e.g., allowing clothing to get wet might result in getting cold).

Finally, according to Hill (2007), WT also integrates concepts from both Adlerian and reality therapies. Adlerian principles within WT include encouragement, holism, and natural consequences (Hill, 2007). Encouragement, for example, is a central feature of facilitating the attainment of self-confidence in WT (Hill, 2007). Moreover, WT integrates concepts from reality therapy (see Glasser, 1998), including concepts of success/failure identities and choice theory (Hill, 2007). Specifically, adolescents seek a success identity; yet, their progress can be hindered by a lack of experience of love and worth (Hill, 2007). The group experiences in WT, however, provide a vehicle through which they can experience feeling worthwhile and valued (Hill, 2007). Also, the initiatives and camping provide needed opportunities to achieve success and integrate this success into their forming identities (Hill, 2007). Overall, these therapies help to explain the client's internal world, i.e., the success/failure identity, and how WT aids the client in generalizing newly learned behavior into normal life through the encouragement of an internal locus of control.

\section{Theoretical models of WT}

Although Gass (1993) is writing about AT programs, he states that there are seven main principles common to programs in North America. First, Gass suggests that WT is an action-centered therapy, meaning that clients are not passive recipients of therapy. Second, Gass explains that WT offers clients an unfamiliar environment, which may foster openness to change since there are fewer expectations about the definition of success. Third, Gass asserts that WT creates a climate of change by providing an optimal level of stress that motivates clients to solve problems through learning more functional behaviors while rewarding them with clear consequences. Fourth, Gass suggests WT provides assessment capabilities. By this, Gass means that the adventure experience provides instructors with rich clinical information about clients as they demonstrate aspects of themselves through problem solving and group behavior. Fifth, Gass maintains that WT offers a group dynamic where clients learn about themselves issues such as self-efficacy and interpersonal impact when they participate in group adventure activities. Sixth, Glass suggests that WT allows clients to focus on their strengths. For example, clients are often well aware of their maladaptive behaviors, but the adventure experience may provide them with opportunities to experience themselves succeed by using their strengths. Finally, WT incorporates the changing role of the therapist by allowing the adventure counselors to actively frame activities in ways that are specific to the issues of the clients (Gass, 1993).

In addition to Gass' (1993) suggestions, a unique aspect of wilderness therapy is its reliance on the outdoors for therapeutic processes (Fletcher \& Hinkle, 2002). WT combines the wilderness setting with psychotherapeutic techniques, helping clients to access complicated feelings and emotions suppressed by anger, depression, or substance abuse (Russell, Hendee, \& Phillips-Miller, 2000). Wilderness therapy also combines both traditional therapeutic interventions such as group or individual therapy with the non-traditional wilderness setting (Hagan, 2003). Fletcher and Hinkle (2002) argue that wilderness therapy is unique in that the actual process of therapy has been taken to an environment that by itself is therapeutic.

\section{Rationale for WT as an alternative treatment}

Today's youth face an increasing avalanche of serious psychosocial problems including drugs, gangs, and a rise in broken or blended families (Bruyere, 2002). Presently, mental health professionals find themselves dealing with a growing range and depth of psychological problems, creating a need for an increasingly diverse and sophisticated range of intervention methodologies (Neill, 2003). A singular intervention strategy may not benefit all youth any more than a singular teaching style will benefit an entire classroom (Bruyere, 2002). Approaches to 
adolescents may need to be especially creative and engaging in order to be effective. For example, traditional and autocratic approaches, such as individual therapy may not be as successful as alternative treatments for delinquent youth (Bruyere, 2002).

Specific benefits of WT include its ability to offer adaptation and flexibility (Sheldon \& Arther, 2001), improve self-efficacy (Williams, 2000), help adolescents learn by doing (Conners, 2000; Russell, 2003; Russell, Hendee, \& Phillips-Miller, 2000), and provide physical activity and risk taking (Berman \& Davis-Berman, 1995). Moreover, in the wilderness, clients can no longer rely on the poor coping skills that may have gotten them by in urban life. Within the solitude of the wilderness, clients may be confronted with their issues and find it difficult to cope, forcing them to expand their available coping resources (SUWS, personal communication, March 22, 2005).

\section{Key differences between WT settings and traditional psychotherapy}

Priest and Gass (1997) propose that WT does not fall under competencies for either outdoor education or traditional psychotherapy due to the unique situation created by psychotherapy implemented in an inherently challenging wilderness environment. Although WT approaches use various aspects of traditional psychotherapy, WT differs significantly from traditional psychotherapy (Fletcher \& Hinkle, 2002) by way of many differing factors including its use of perceived risk (Fletcher \& Hinkle, 2002; Russell, 2003), assisting clients in providing their own linkages, bridges, and connections to what they are learning so that they can integrate their personal insights and new behaviors into their new lifestyle at home (Nadler \& Luckner, 1992), the use of narrative (Epston et al., 1996), the use of metaphor (Adler, 1957; Stoufer, 1999), the use of isolation (Russell \& Phillips-Miller, 2002), and building skills such as problem solving, decision analysis, improved coping, and other abilities that foster independence and greater self-efficacy (Ferguson, 1999).

Wilderness therapies may also require traditional counseling skills (e.g., soft skills), as well as additional outdoor physical abilities (e.g., hard skills)(Fletcher \& Hinkle, 2002). Soft skills such as reflective listening, problem solving, leadership, reframing, and communication are basic necessary components of any therapy, whereas hard skills such as climbing or kayaking must be integrated in order for adventure therapy to take place.

An additional consideration in the differences between WT and traditional psychotherapy is that much of the business from WT programs comes from referrals provided by educational consultants (personal communication, SUWS 2005). WT psychologists often work closely with educational consultants as supportive members of the treatment team. WT psychologists must familiarize themselves with the expectations and needs of these consultants in order to improve cohesion of the treatment team. Creating a working relationship among the various members of the treatment team is important for the smooth transition of clients between organizations and aftercare. WT psychologists also need to educate the consultants about the process that occurs throughout treatment, as well as give updates on the client's progress in the program. These consultants often make critical referrals and recommendations for continued care based on input from the psychologist. Knowledge of how to work with educational consultants impacts both the transition into WT programs and helps to facilitate proper aftercare for clients (personal communication, SUWS 2005).

Another key difference in WT is that the therapists may actually live with their clients (Russell \& Phillips-Miller, 2002). This variable adds significant complications to the boundaries for typical therapeutic relationships. However, one benefit may be that it may allow clients to form therapeutic rapport more quickly with leaders. Clients may also get a chance to observe the therapists in real-life situations and challenges, making leaders more approachable than in traditional settings (SUWS personal contact, March 22, 2005). However, this predicament may also create significant difficulty for therapists entering the field from more traditional mental health settings.

Moreover, clients in WT programs may need more support than clients in a traditional therapy setting. In WT, clients are placed in an unpredictable environment often far removed from family and friends. For some youth, this is the first time that they have been away from home for an extended period of time (Russell, Hendee, \& Phillips-Miller, 2000). Because this variable is not traditionally present in therapy, the therapist must examine how this may impact the course of treatment.

Furthermore, assessment is largely absent from the literature in WT. Effective assessment is important because psychologists need to make informed decisions about the suitability of interventions. Suitability may be determined by previously attempted solutions, attainable goals, and client-specific considerations. Taking time in the process of assessment may drastically change the efficacy of the interventions in WT. The field of psychological consultation considers assessment to be one of the most important steps in therapeutic change, and should perhaps consist of as much as two-thirds of the therapeutic process (Caplan, 1970). Because of this, a 
focus on effective assessment may be another critical area for future WT research. Ultimately, professionals in WT need to be competent in assessment skills in order to benefit the clients they serve.

Lastly, the literature suggests that proper follow-up interventions for home environment (e.g., parents, churches, group homes) may prove to be the most effective agent for sustaining changes brought about during WT (Bruyere, 2002). The importance of this finding cannot be overstated given its implications for sustaining therapeutic change. WT programs may benefit by including interventions for the family system or adult support networks of adolescent or adult clients (Bruyere, 2002). Teaching interventions to the broader support network may ensure lasting change and allow families to effectively cope with and respond to mental health clients.

\section{Supportive findings for WT}

Numerous studies suggest that wilderness and adventure based therapy is effective in treating a range of issues relevant to mental health with a variety of age groups and populations (Bedard, 2005; Clark, Marmol, Cooley, \& Gathercoal, 2004; Hattie, Marsh, Neill, \& Richards, 1997; Nadler \& Luckner, 1992; Neill, 2003; Wilson \& Lipsey, 2000) For instance, Wilson and Lipsey conducted a meta-analysis of 28 studies that utilized a control group in the evaluation of the effectiveness of wilderness challenge programs for delinquent adolescents between the ages of 10 to 18 . The overall mean effect size was 0.18 for delinquency outcomes, which equals a recidivism rate of $29 \%$ for wilderness program participants and $37 \%$ for the control group. Hattie et al. (1997) conducted a meta-analysis of 96 studies examining the effects of adventure education for participants ages 11 to 42 on six outcome categories: self concept, leadership, academic, personality, interpersonal, and adventuresomeness, revealing an overall mean effect size of 0.34. Finally, Bedard (2005) conducted a meta-analysis examining the effects of wilderness therapy with adolescent delinquents between the ages of 10 and 18 on three different outcomes: behavior change/interpersonal skills, self-esteem/self-concept, and recidivism. Regarding behavior change/interpersonal skills, eight studies yielded an effect size of 0.50 . In terms of self-esteem/self-concept, 16 studies generated an effect size of 0.53 . The 13 studies that measured recidivism generated an effect size of 0.30 . Finally, Clark et al. (2004) examined the effectiveness of a wilderness therapy program with 109 adolescents between the ages of 13 and 18 . Results revealed significant reductions in the clinical syndrome, personality pattern, and expressed concerns scales of the Millon Adolescent Clinical Inventory (MACI).

Moreover, Davis-Berman and Berman (1994) note that WT appears to be a treatment of choice for adolescents. In agreement, Williams (2000) argues that adventure therapy is one of the most popular treatments for adolescents. Additionally, based on a review of the literature, Russell (2000) found that WT improves self-perceptions, increases social adjustment, and reduces rates of recidivism.

WT has also been shown to increase anger management skills, responsibility, communication, sharing, assertiveness, interpersonal skills, and trust in male victims (ages 9 to 11) of abuse and neglect (Mcnamara, 2002). In addition, Russell and Phillips-Miller (2002) note that adolescents in WT programs spend less time talking about "war stories" and more time focusing on clinical issues. Furthermore, evidence suggests that there is a positive correlation between WT program length and overall lasting behavioral change (Neill, 2003).

What is more, WT has been shown to be an alternative to psychiatric hospitalization (see Berman \& Anton, 1989; Berman \& Davis-Berman, 1994). Also, authors such as Nassar-Mcmillan and Cashwell (1997), Martinez (2003), Neill (2003), and Hans (2000) suggest that WT is effective because it enhances self-esteem in youth, has a positive effect on the ability to internalize locus of control, and allows clients to perceive themselves as more capable of influencing their own lives.

WT has also been empirically validated with special populations, such as those in family counseling, those struggling with female and male body image, disabled populations, college students, corporate teams, victims of trauma and physical illness, athletes, bipolar disorder populations, cerebral palsy populations, mentally retarded populations, speech impairment populations, traumatic brain injury populations, Christian populations, and psychotic populations (Anderson, Schlein, McAvoy, Lais, \& Seligman, 1997; Fletcher \& Hinkle, 2002; Griffin, 2003; Pawlowski, Holme, \& Hafner, 1993).

\section{Movement toward standards of competence}

There is an increasing demand from consumers, workers, insurance companies, government, and the general public for mental health interventions to be accountable for their claims (Neill, 2003). Because of this, interventions such as WT need to deliver measurable and positive impacts on both clients and society. WT's lack of an industry standard in competence could lead to a diffusion of treatment. Professional competence is extremely important for advancement and recognition for the field of WT. The current differences in training, experience, and techniques used by different staff members make valid comparisons across WT programs quite 
challenging (Newes, 2001). By holding these variables constant, there can be an increase in the likelihood that services will be delivered in a more consistent fashion. Furthermore, consistency in established treatment delivery should bring about greater success rates for WT programs.

Ultimately, the literature suggests that the field of WT lacks a current standard of delivery, making objective statements about standards of care, leadership, quality of service, effective interventions, and principles of treatment very difficult. As Russell (2003) suggests, new standards shape the field from the top down. It is dually hoped that standards of competency for WT psychologists will help to unify the field, which it will need in order to gain respect and integrity.

\section{Proposed competencies}

It is incumbent upon psychologists working in new competency arenas to promote a more complete understanding of what constitutes effectiveness, particularly when sustained behavioral change is the desired outcome (Brotman, Liberi, \& Wasylyshyn, 1998). As new areas of psychological practice emerge, they are eventually subjected to the ethical scrutiny of the field in order to ensure that professional psychologists in all areas are accurately representing their knowledge and skills. In keeping with the way that the field has historically distinguished specific areas, this article has drawn upon previous specialty models in psychology to create a frame of reference for proposed core competencies in WT. Although a comprehensive exploration of each specialty area is beyond the scope of this article, a brief summary of the three specialty areas the WT competencies are drawn from is appropriate.

One of the specialties acknowledged by the APA is that of consulting psychology. Gerald Caplan, a figurehead in the field of consulting psychology, projected that consultation and executive coaching (EC) was not a modified form of therapy but its own discipline (Caplan, Caplan, \& Erchul, 1995). This foreshadowed the eventual recognition of executive coaching (EC) and consulting psychology as a specialty area, which now merits its own APA journal. Today, consulting psychology exists as a specialty area acknowledged by the APA.

In addition to consulting psychology, the American Psychological Association (APA) published an article in 1994 laying down guidelines for competence in child custody evaluations. These guidelines are built upon the APA code of ethical conduct from 1992 and are designed to promote proficiency in the use of psychological expertise in child custody evaluations. The APA proposes that psychologists must be able to demonstrate a clear sense of direction and purpose in conducting the evaluations and to perform their roles ethically (APA, 1994). Based on ethical concerns, the APA (1994) delineates 16 competencies to aid in the ethical practice of custody evaluations by psychologists to increase the fairness of the determination that the court must make.

A third major area that has been recognized as a specialized competency is forensic psychology (Committee on Ethical Guidelines for Forensic Psychology, 1991). Forensic psychology was a forerunner of specialization due to its extensive interaction with the legal system. It soon became obvious that psychologists would need to have an understanding of the law in order to work effectively as a Forensic Psychologist. Principle $2.01 \mathrm{f}$ of the APA Ethics code (2003) states, "When assuming forensic roles, psychologists are to become reasonably familiar with the judicial system or administrative roles governing their rules." Forensic psychology is now recognized as a specialty competency due to its extensive involvement with courts, legal proceedings, correctional and forensic mental health facilities, and legislative agencies, which requires additional training on the part of the clinical psychologist.

While informed by the Ethical Principles of Psychologists (APA, 2003), and meant to be consistent with them, the following guidelines for WT are designed to provide more specific guidance to WT psychologists in monitoring their professional conduct when acting as a psychologist for WT programs. By design, none of the guidelines contradict any of the principles of the ethical code, but rather amplify those principles in the context of the practice of WT. The primary goal is to improve the quality of WT psychological services offered to individual clients, their families, and WT programs to thereby enhance WT as a discipline and profession. Upon being hired by a licensed WT program, it is suggested that each new psychologist be put through an orientation process similar to that of other staff members to become acquainted with principles of the WT atmosphere and program. The competencies proposed here are intended to elaborate on general competencies for all staff (Accreditation Council of the Association for Experiential Education, 2005, Chapter 3), as they are specifically intended for psychologists. The guidelines specify the nature of desirable professional practice for psychologists working in the wilderness setting.

As guidelines, they are aspirational in nature and not intended to be mandatory or exhaustive. The goal of these guidelines is to promote proficiency in using psychological expertise (APA, 1994) in the field of WT. It is hoped that they may serve as an impetus for improved treatment and a foundational guide for future WT psychologists. 
Through analysis of the literature and by adopting APA's (2003) ethical principles as a template, several key competencies have emerged in delineating the specialized role of the WT clinician. Table I elucidates many key competencies necessary to allow for standardization of WT.

\section{Knowledge of experiential learning models and philosophical foundations}

While experiential learning models vary from theorist to theorist, it is generally agreed upon that there are four distinct phases that comprise the learning cycle of experiential education: experience, reflection, processing, and application (Nadler \& Luckner, 1992). Experiential Education posits that experience occurs in all life situations, but experiential learning involves intentionally planning and implementing new behaviors. Thus, the structured experience is the stage for learning.

Generally, clients are encouraged to experiment with new behaviors during the first phase. In this phase, clients have the opportunity to observe themselves using these new behavioral skills in the moment. It is hoped that they will experience satisfaction over the success of utilizing the new skill and thus integrate it into their existing repertoire of behaviors. In programs designed for delinquent youth, instructors reward adaptive behavior. When delinquent youth enter a WT program, they often begin by using the maladaptive behaviors that they are comfortable using. However, when they get a chance to reflect on themselves using these behaviors over and over again without progressing in the programs, it is theorized that they will be more open to experimenting with alternative and more adaptive behavior.

The event of processing plays a critical role in helping to reinforce some of these new skills into the clients' existing repertoire (1992). The processing phase refers to a time when the clients can reflect, describe, analyze, and communicate exactly what it is that they have experienced (1992). This phase constitutes the cornerstone of an adventure learning experience.

Finally, WT psychologists should be familiar with common integrative concepts that provide a framework for wilderness therapy, such as reality therapy (see Glasser, 1998), in order to conceptualize the inner life of clients and how they generalize their newly learned behavior (Hill, 2007). Specifically, adolescents are naturally seeking a success identity, although their progress in this regard may be hindered by the lack of experience of love and worth (Hill, 2007). Thus, WT group experiences help adolescents to internalize and crystallize feelings of being worthwhile and valued, which strengthens their sense of self. Finally, the WT initiates help clients to find success and generate an internal locus of control, which helps them to generalize the newly learned behaviors (Hill, 2007).

\section{Sensitivity to the nature of a new environment}

How clients come to be in WT programs can be very different from traditional psychotherapy clients. Many WT programs are used as threats by parents, alternative punishment, or even preventative alternatives to residential or institutional placement. Many clients are ordered or coerced into participation in a program against their own free will (Hunt, 1994). Coercing or forcing a client into 45 days of therapy in another region of the U.S. may be more intense than pushing them into a 45 minute therapy session. Thus, many clients enter programs in an emotionally chaotic status that may exceed that of the traditional therapeutic setting.

The WT psychologist should be aware that many clients enter the program with a great deal of fear and anxiety regarding what the program might be like. On the other hand, many clients enter programs with a great deal of anger and resistance. Since motivation for change is often low, WT psychologists should familiarize themselves with literature geared towards this population.

\section{The use of real or perceived risk}

Risk is an inherent part of the adventure and wilderness environment (Hunt, 1994). Adventure education involves putting clients in potentially harmful situations. This inherent risk brings a different element to WT, making it distinctly different from traditional psychotherapy. Although some might consider risk to be controversial since it involves inciting fear in clients, it may create a disrupted internal state necessary to catalyze change. A new WT psychologist should be familiar with the risks inherent in conducting therapy in the wilderness environment as well as the variables that risk might produce. Because risk or perceived adventure is the essence of many wilderness programs, theoretically, clients should be more vulnerable or open to change in behavior during this period of relative instability. Moreover, due to this perceived risk, clients may react in unpredictable or extreme ways to escape the anxiety that this new environment creates. The WT clinician should be well equipped to respond with the appropriate level of support, particularly with regard to suicidal ideation. 


\section{Use of extensive intake assessment procedures}

The psychologist is the member of the team responsible for admitting new clients to a program. Although careful intake procedures are important in any setting, extensive screening is of particular importance in wilderness settings since clients may have health conditions that could be exacerbated by an outdoor or adventure environment. They must take every step to ensure that they have gathered as thorough a medical and psychiatric history as possible so that they can be aware of medical or medication complications influenced by the wilderness setting. Furthermore, since suicide is a serious risk in adolescents with emotional disturbances, the WT psychologist must be sure to gather extensive history of suicidal ideation and attempts, and to be aware of how the wilderness setting may impact these issues. The WT psychologist should understand the program protocol for how to respond to clients that run away or attempt suicide in this environment. The WT psychologist is obligated to take all the necessary measures to ensure the physical and psychological safety of the client and to educate other staff members of potential risks (Accreditation Council of the Association for Experiential Education, 2005, p. 23).

\section{Supervision skills}

Many WT psychologists visit their clients in the field several times per week for sessions. The psychologist will often drive out to the field site to meet with multiple clients at a given camp (SUWS, personal communication, March 22, 2005). During this time, the psychologist should get feedback from field instructors about a given client's progress in the program or behavior since the last session.

Moreover, field instructors frequently have trouble with specific clients. During this time, they often seek direction from the psychologist about how to intervene or redirect the clients' maladaptive behavior. Since field instructors are with clients 24 hours a day, it is critical that they work as a team with the staff psychologist. All members of the treatment team should be clear about the overall goals of each client so that they can work in conjunction with one another. The WT psychologist must be able to think and respond with appropriate interventions that are practical, attainable, and realistically able to be implemented by field instructors who lack exhaustive knowledge of psychology.

\section{Dual relationships}

The WT psychologist should be aware of the difficulties potentially posed by dual roles inherent in wilderness or adventure settings, and should conduct himself or herself with the utmost regard for therapeutic boundaries. Specifically, dual relationships may be difficult to negotiate clearly when a psychologist actually lives with the client. This may be the most blatant example of potential boundary problems. It may become more difficult in this instance to maintain a clearly defined role in the client's life.

\section{Consultation skills}

WT psychologists need to show skills in consultation. Psychologists who work within WT programs must have the diplomatic savvy to work with educational consultants, families, and other organizations to facilitate the best possible treatment for the clients. In the WT setting, it is often necessary to report the client's progress to residential facilities and other entities. At this time, psychologists must be skilled in effectively communicating progress and educating about aftercare and necessary follow-up procedures. Psychologists fulfill a critical role in facilitating a smooth transition for clients entering and graduating from WT programs.

\section{Therapeutic contract and termination}

Issues surrounding therapeutic contracting and termination are particularly important since clients in WT programs may not have the option of continuing therapy with the therapist. For example, clients often graduate from programs after unspecified lengths of time and there may not be sufficient time to address issues in depth due to a forced closure to therapy. For this reason, WT psychologists should take every step to ensure that the clients are clearly informed about the nature of the therapeutic contract and potential aftercare referrals.

\section{Abuse reporting and clients rights}

Since psychologists in WT programs serve as confidants and trusted figures to clients in WT programs, they are often the first to hear about allegations of abuse. It is paramount that psychologists know the process of how to report potential abuse by peers and colleagues such as field instructors. Agencies should have a protocol for this since it is not uncommon for emotionally disturbed adolescents to make accusations about field instructors. Sadly, actual abuse may take place on occasion. However, it may place the psychologist in a very awkward position since it may be quite obvious that the client reported the psychologist's peer, which can make for difficult working conditions. It is equally important that the WT psychologist knows how to go about reporting 
abuse across state lines since many clients do not live in the same state as the program. This process should be clearly delineated prior to beginning fieldwork.

Although it is suggested that it is not the responsibility of the psychologist to investigate abuse claims, but simply to report allegations, abuse can be looked upon harshly by programs or staff members looking to preserve a reputation. Although psychologists are not formal client advocates, the WT psychologist should act with the utmost integrity and ethical judgment with regard to the client's best interests and should be prepared to assist clients in knowing and maintaining their rights. Additionally, psychologists are often more aware of ethical obligations as mandated reporters and can serve as educators to other staff members regarding the importance of advocating for patients and reporting alleged abuse or neglect.

\section{Respect for the client's rights and dignity}

Consistent with the General Principles of APA (2004), WT psychologists respect the dignity, worth, and individuality of every client. WT psychologists respect cultural, individual, and role differences, including those based on age, gender, gender identity, race, ethnicity, culture, national origin, religion, sexual orientation, disability, language, and socioeconomic status. WT psychologists consider the above factors when working with those participating in WT. Finally, WT psychologists attempt to eliminate possible effect these biases may have on their work, and do not condone such biases on the part of others.

\section{Conclusion}

WT has been identified as an emerging competency in the practice of psychology. The literature has revealed a gap in standards of current practice for WT psychologists. In spite of this, psychologists are increasingly being hired by WT programs for their expertise in sustained behavioral modification. Moreover, psychologists may be increasingly sought out as WT staff members in order for WT organizations to be recognized by managed care as possessing a high standard of mental health treatment.

In the absence of a credentialing process for new WT psychologists, the field needs defined standards for what it means to be a WT psychologist. The proposed core competencies can provide excellent information to new WT psychologists inquiring about what differences are posed by the wilderness setting. Furthermore, the proposed competencies can provide clarification to WT programs who hire new psychologists and families who desire an objective standard by which to compare or evaluate the professionals with whom they may entrust their children. As noted earlier, any movement toward a new field of specialization should ultimately be based on the public need (APA, 2000). Of equal importance, the goal of this article was also to improve the quality of ethical practice for psychologists in this burgeoning young field of WT. The new standards proposed in this article may begin the process of setting a higher standard of practice for WT psychologists and provide additional credibility and legitimacy to a field that is gaining much attention.

The next step may be to implement training or credentialing programs to facilitate a specialization for WT psychologists. In addition, future studies may wish to focus on specific training models. For the time being, however, psychologists may need to consult with existing training programs for wilderness or adventure therapy (see Puchbauer, 2006). WT psychologists may also seek training manuals such as Puchbauer (2006) to build a base of skills.

Only through established guidelines for competency can this new field truly thrive. It is hoped that the proposed model will equip future WT psychologists with the requisite knowledge and skills necessary to begin at a higher level of ethically competent practice. It is dually hoped that, through their experience, future psychologists will continue to improve upon and add to these guidelines.

\section{References}

American Psychological Association. (1994). Guidelines for child custody evaluations in divorce proceedings. American Psychologist. 49, 677-680.

American Psychological Association. (2000). Petition for the recognition of a specialty in professional psychology. American Psychological Association. Washington, DC

American Psychological Association. (2003). Code of ethics in professional practice of psychology. American Psychological Association. Washington, DC

Aspen Academy. (2006, September 1). [Online] Available: http:/www.aspenacademy.com. (September 1, 2006)

Bandoroff, S. (1992). Wilderness family therapy: An innovative treatment approach for problem youth. Unpublished doctoral dissertation, University of South Carolina. 
Bedard, R. (2005). Wilderness therapy programs for juvenile delinquents: A meta-analysis. Dissertation. Colorado State University.

Berman, D., \& Davis-Berman, J. (1995). Lifestories: Discussions on death using adventure-based activities. Activities, Adaptation, and Aging. 19, 55-63.

Berman, D., \& Davis-Berman, J. (2001). Critical and emerging issues for the therapeutic adventure. Journal of Experiential Education. 24, 68-69.

Brotman, L., Liberi, W., \& Wasylyshyn, K. (1998). Executive Coaching: The need for standards of competence. Consulting Psychology Journal: Practice and Research, 50, 40-46.

Bruyere, B. (2002). Appropriate benefits for outdoor programs targeting juvenile male offenders. Journal of Experiential Education. 25, 207-213.

Caplan, G. (1970). The Theory and Practice of Mental Health Consultation. New York, New York: Basic Books Inc.

Clark, J., Marmol, L., Cooley, R., \& Gathercoal, K. (2004). The effects of wilderness therapy on the clinical concerns (on axes I, II, and IV) of troubled adolescents. Journal of Experiential Education, 27, 213-232.

Committee on Ethical Guidelines for Forensic Psychologists. (1991). Specialty guidelines for forensic psychologists. Law and Human Behavior, 15.

Davis-Berman, J., \& Berman, D. (1994). Wilderness therapy. Dubuque, Iowa: Kendell Hunt Publishing.

Fletcher, T., \& Hinkle, J. (2002). Adventure Based Counseling: An Innovation in Counseling. Journal of Counseling and Development, 80, 277-285

Friese, G., Hendee, J., \& Kinziger, M. (1998). The wilderness experience program industry in the United States: Characteristics and dynamics. Journal of Experiential Education, 21, 40.

Gass, M. (1993). Foundations of adventure therapy. In M. Gass (Ed.), Adventure therapy: Therapeutic applications of adventure programming (p. 3-10). Dubuque, IA: Kendell/Hunt.

Gillis, H., \& Casson, D. (1994). A meta-analysis of outdoor adventure programming with adolescents. Journal of Experiential Education, 17, 40-50.

Glasser, W. (1998). Choice theory: A new psychology of personal freedom. New York: HarperCollins Publishers, Inc.

Griffin, J. (2003). The effects of adventure based programming with an explicit spiritual component on the spiritual growth of adolescents. Journal of Experiential Education, 25, 351.

Hagan, J. (2003). An alternative therapy for the behaviorally challenged youth: The efficacy of wilderness therapy programs. Dissertation. The University of Toledo.

Hans, T. (1997). A meta-analysis of the effects of adventure programming on locus of control. Unpublished master's thesis, Georgia College, Milledgeville, GA.

Hans, T. (2000). A meta-analysis of the effects of adventure programming on locus of control. Journal of Contemporary Psychotherapy, 30, 33-60.

Hattie, J., Marsh, H., Niell, J., \& Richards, G. (1997). Adventure education and Outward Bound: Out of class experiences that make a lasting difference. Review of Educational Research, 67, 43-87.

Hill, N. (2007). Wilderness therapy as a treatment modality for at-risk youth: A primer for mental health counselors. Journal of Mental Health Counseling, 29, 338-349.

Hunt, J. (2002). Ethical Issues in Experiential Education. The Association for Experiential Education. Dubuque, Iowa: Kendal/Hunt Publishing.

Leemon, D., Place, S., Ajango, D., \& Wood, H. (2005). Manual of Accreditation Standards for Adventure Programs, Fourth Edition. Boulder, CO: Association for Experiential Education.

Marsh, P. (1999). What does camp do for kids? A meta-analysis of the influence of the organized camping experience on the self constructs of youth. Unpublished master's thesis, Indian University, Martinsville, IN.

Martinez, M. (2002). A wilderness therapy program for a diverse group of at risk adolescent boys: Changes in self-esteem and locus of control and their relationship to group affiliation. Dissertation. Alliant University. San Francisco, CA. 
Mcnamara, D. (2002). Adventure-based programming: Analysis of therapeutic benefits with children of abuse and neglect. Adolescence, 62, 730.

Nadler, R., \& Luckner, J. (1992). Processing the Adventure Experience: Theory and Practice. Kendall Hunt Publishing: Dubuque, Iowa.

Nassar-McMillan, S., \& Cashwell, C. (1997). Building self-esteem of children and adolescents through adventure-based counseling. Journal of Humanistic Counseling Education \& Development, 36, 59-67.

Neill, J. (2003). Reviewing and Benchmarking Adventure Therapy Outcomes: Applications of Meta-Analysis. Journal of Experiential Education. 25, 316-321.

Newes, S. (2001). Future Directions in Adventure-Based Counseling Research: Methodological Considerations and Suggestions. Journal of Experiential Education, 24, 92-99.

Priest, S. (2001). A program evaluation primer. Journal of Experiential Education. 24,34-40.

Priest, S., \& Gass, M. (1997). Effective leadership in adventure programming. Champaign, IL: Human Kinetics.

Puchbauer, D. (2006). Outdoor Behavioral Healthcare for troubled adolescents: A clinical skills training manual for adventure counselors. Unpublished Doctoral Dissertation. Azusa Pacific University.

Roe, R. (2002). What makes a competent psychologist? European Psychologist. 7, 192-202.

Russell, K. (2000). Exploring how wilderness therapy process relates to outcomes. Journal of Experiential Education. 23, 170.

Russell, K. (2001). Assessment of treatment outcomes in outdoor behavioral healthcare (Tech. Rep. No. 28). Moscow, ID: Idaho Forest, Wildlife and Range Experiment Station.

Russell, K. (2003). A nation-wide survey of outdoor behavioral healthcare programs for adolescents with behavior problems. Journal of Experiential Education, 25, 322-331.

Russell, K., \& Phillips-Miller, D. (2002). Perspectives on the wilderness therapy process and its relation to outcome. Child \& Youth Care Forum, 31, 415-437.

Russell, K., Hendee, J., \& Phillips-Miller, D. (2000). How Wilderness Therapy Works: An Examination of the Wilderness Therapy Process to Treat Adolescents with Behavioral Problems and Addictions. USDA Forest Service Proceedings, RMRS, 3, 15.

Schoel, J., Prouty, D., \& Radcliffe, P. (1988). Islands of healing: A guide to adventure-based counseling. Hamilton, Maine: Project Adventure, Inc.

Sheldon, N., \& Arthur, N. (2001). Adding Adventure to Therapy. Guidance \& Counseling, 16, 67.

Sugerman, D. (2000). Adventure experiences for older adults: Examining current practices. Journal of Experiential Education, 23, 12.

Williams, B. (2000). The treatment of adolescent populations: An institutional vs. a wilderness setting. Journal of Child \& Adolescent Group Therapy, 10, 47-56.

Wilson, S., \& Lipsey, M. (2000). Wilderness challenge programs for delinquent youth: A meta-analysis of outcome evaluations. Evaluation and Program Planning, 23, 1-12. 
Table I. Proposed Core Competencies for Wilderness Psychologists

\begin{tabular}{|c|c|}
\hline Principle & Description \\
\hline $\begin{array}{l}\text { 1. Demonstrates knowledge of } \\
\text { experiential learning models and } \\
\text { adventure processing. }\end{array}$ & $\begin{array}{l}\text { The WT psychologist makes reasonable efforts, prior to beginning practice in WT, } \\
\text { to become familiar with experiential learning models and theories of adventure } \\
\text { processing since these models are largely absent from clinical psychology curricula. } \\
\text { This includes philosophical foundations of WT that offer frameworks for } \\
\text { understanding changes that take place within the client's internal world and how the } \\
\text { client generalizes newly learned behavior into normal life. }\end{array}$ \\
\hline $\begin{array}{l}\text { 2. Is sensitive to the nature of a new } \\
\text { environment for the client. }\end{array}$ & $\begin{array}{l}\text { The WT clinician understands that the WT environment often creates a disrupted } \\
\text { internal state for the clients who find themselves in an environment far removed } \\
\text { from home with the understanding that many clients are forced into treatment. }\end{array}$ \\
\hline $\begin{array}{l}\text { 3. Is aware of the potential impact of } \\
\text { utilizing real or perceived risk in } \\
\text { therapy. }\end{array}$ & $\begin{array}{l}\text { The WT psychologist understands that WT differs considerably from traditional } \\
\text { psychotherapy in that it is often conducted in an unpredictable outdoor environment } \\
\text { and frequently includes an intentional component of real or perceived risk. The WT } \\
\text { psychologist endeavors to understand the consequent impact of risk on the physical } \\
\text { and psychological safety of the client. }\end{array}$ \\
\hline $\begin{array}{l}\text { 4. Uses extensive intake assessment } \\
\text { procedures. }\end{array}$ & $\begin{array}{l}\text { The WT psychologist conducts an extensive intake assessment with regard to } \\
\text { critical issues such as health problems or risk of suicide that could be particularly } \\
\text { impacted by the wilderness setting. }\end{array}$ \\
\hline $\begin{array}{l}\text { 5. Is able to exercise supervision } \\
\text { skills. }\end{array}$ & $\begin{array}{l}\text { The WT clinician can provide effective supervision and suggestions for intervention } \\
\text { to field supervisors who often live with clients } 24 \text { hours a day. }\end{array}$ \\
\hline $\begin{array}{l}\text { 6. Recognizes how dual relationships } \\
\text { are impacted by the wilderness } \\
\text { setting. }\end{array}$ & $\begin{array}{l}\text { The WT psychologist is aware of the difficulties potentially posed by dual roles } \\
\text { sometimes present in wilderness or adventure settings, and conducts himself or } \\
\text { herself with the utmost regard for therapeutic boundaries and the client's best } \\
\text { interests. }\end{array}$ \\
\hline 7. Demonstrates consultation skills. & $\begin{array}{l}\text { The WT clinician demonstrates the ability to consult with educational consultants, } \\
\text { courts, families, and other organizations. This skill is critical to facilitating a smooth } \\
\text { transition for clients entering and graduating from programs. }\end{array}$ \\
\hline $\begin{array}{l}\text { 8. Recognizes how the therapeutic } \\
\text { contract and termination are impacted } \\
\text { by the WT setting. }\end{array}$ & $\begin{array}{l}\text { WT psychologists should take every precaution to prepare clients for termination } \\
\text { and acquire adequate training for how clients' rights are impacted by the wilderness } \\
\text { setting. }\end{array}$ \\
\hline $\begin{array}{l}\text { 9. Recognizes how abuse reporting } \\
\text { and clients rights are impacted by the } \\
\text { wilderness setting. }\end{array}$ & $\begin{array}{l}\text { Because psychologists in WT programs serve as a confidant and trusted figure to the } \\
\text { client in WT programs, they may be first to hear about allegations of abuse in a } \\
\text { program. The WT psychologist is aware of the exact protocol with regard to } \\
\text { reporting alleged abuse out-of-state or within program. }\end{array}$ \\
\hline $\begin{array}{l}\text { 10. Recognize the individual rights } \\
\text { and dignity of each client. }\end{array}$ & $\begin{array}{l}\text { WT psychologists respect the dignity, worth, and individuality of every client. WT } \\
\text { psychologists respect cultural, individual, and role differences, including those } \\
\text { based on age, gender, gender identity, race, ethnicity, culture, national origin, } \\
\text { religion, sexual orientation, disability, language, and socioeconomic status. }\end{array}$ \\
\hline
\end{tabular}

